\title{
Physics Today
}

\section{Dick Feynman-The Guy in the Office Down the Hall}

Murray Gell-Mann

Citation: Physics Today 42(2), 50 (1989); doi: 10.1063/1.881192

View online: http://dx.doi.org/10.1063/1.881192

View Table of Contents:

http://scitation.aip.org/content/aip/magazine/physicstoday/42/2?ver=pdfcov

Published by the AIP Publishing

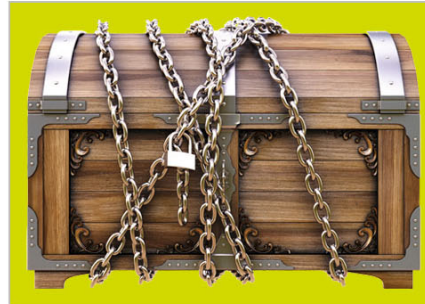

- Visit www.knfusa.com/noescape 


\title{
DICK FEYNMAN-THE GUY IN THE OFFICE DOWN THE HALL
}

\author{
A brilliant, vital and amusing neighbor, Feynman was \\ a stimulating (if sometimes exasperating) partner in discussions \\ of profound issues. His sum-over-paths method may turn out \\ to be not just useful, but fundamental.
}

\author{
Murray Gell-Mann
}

\begin{abstract}
I hope someday to write a lengthy piece about Richard Feynman as I knew him (for nearly 40 years, 33 of them as his colleague at Caltech), about our conversations on the fundamental laws of physics, and about the significance of the part of his work that bears on those laws. In this brief note, I restrict myself to a few remarks and I hardly touch on the content of our conversations.
\end{abstract}

When I think of Richard, I often recall a chilly afternoon in Altadena shortly after his marriage to the charming Gweneth. My late wife, Margaret, and I had returned in September 1960 from a year in Paris, London and East Africa; Richard had greeted me with the news that he was "catching up with me"- he too was to have an English wife and a small brown dog. The wedding soon took place, and it was a delightful occasion. We also met the dog (called Venus, I believe) and found that Richard was going overboard teaching her tricks (leading his mother, Lucille, with her dry wit, to wonder aloud what would become of a child if one came along). The Feynmans and we both bought houses in Altadena, and on the afternoon in question Margaret and I were visiting their place.

Richard started to make a fire, crumpling up pages of a newspaper and tossing them into the fireplace for kindling. Anyone else would have done the same, but the way he made a game out of it and the enthusiasm that he poured into that game were special and magical. Meanwhile, he had the dog racing around the house, up and down the stairs, and he was calling happily to Gweneth.

Murray Gell-Mann is Robert A. Millikan Professor of Theoretical Physics at the California Institute of Technology.
He was a picture of energy, vitality and playfulness. That was Richard at his best.

He often worked on theoretical physics in the same way, with zest and humor. When we were together discussing physics, we would exchange ideas and silly jokes in between bouts of mathematical calculation-we struck sparks off each other, and it was exhilarating.

What I always liked about Richard's style was the lack of pomposity in his presentation. I was tired of theorists who dressed up their work in fancy mathematical language or invented pretentious frameworks for their sometimes rather modest contributions. Richard's ideas, often powerful, ingenious and original, were presented in a straightforward manner that I found refreshing.

I was less impressed with another well-known aspect of Richard's style. He surrounded himself with a cloud of myth, and he spent a great deal of time and energy generating anecdotes about himself.

Sometimes it did not require a great deal of effort. For example, during my first decade at Caltech there was a rule at our faculty club, the Athenaeum, that men had to wear jackets and ties at lunch. Richard usually came to work quite conventionally dressed (for those days) and hung his jacket and tie in his office. He rarely ate lunch at the Athenaeum, but when he did, he would often make a point of walking over in his shirt sleeves, tieless, and then putting on one of the ragged sport coats and one of the loud ties that the Athenaeum provided in the cloakroom for men who arrived unsuitably attired.

Many of the anecdotes arose, of course, through the stories Richard told, of which he was generally the hero, and in which he had to come out, if possible, looking smarter than anyone else. I must confess that as the years went by I became uncomfortable with the feeling of being a 


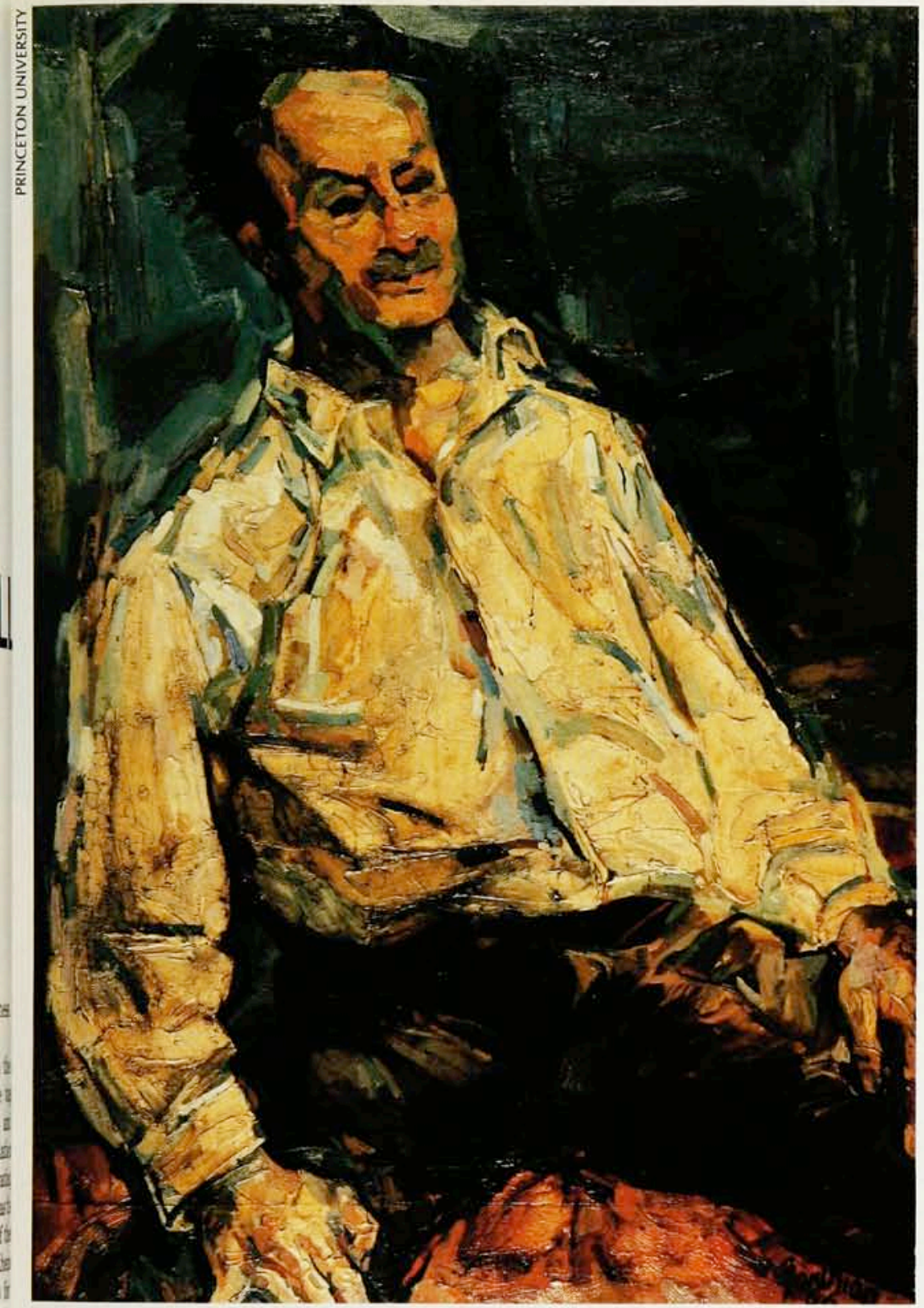

Portrait of Richard Feynman by Jirayr Zorthian, one of Feynman's art teachers. The painting hangs in Jadwin Hall at Princeton University. rival whom he wanted to surpass; and I found working with him less congenial because he seemed to be thinking more in terms of "you" and "me" than "us." Probably it was difficult for him to get used to collaborating with someone who was not just a foil for his own ideas (especially someone like me, since I thought of Richard as a splendid person to bounce my ideas off!).

At first, none of that was much of a problem, and we had many fine discussions in those days. In the course of those talks not only did we "twist the tail of the cosmos," but we also exchanged a good many lively reminiscences about our experiences in research

\section{Summing over histories}

He told me, of course, of his graduate student days at Princeton and his adventures with his adviser, John Wheeler. Wheeler judged their work on the "absorber theory of radiation" to be too much of a collaboration to qualify as a dissertation for the $\mathrm{PhD}$, and so Richard pursued his interest in Paul Dirac's work on the role of the action $S$ in quantum mechanics. In his book on quantum mechanics, and even more in his article in the Physikalische Zeitschrift der Sowjetunion in 1932, Dirac had carried the idea quite far. He had effectively shown how a quantum mechanical amplitude for the transition from a set of values of the coordinates at one time to another set of values at a later time could be represented as a multiple integral, over the values of the coordinates at closely spaced intermediate times, of $\exp (\mathrm{i} S / \hbar)$, where $S$ is the value of the classical action along each sequence of intermediate coordinate values. What Dirac had not done was to state the result in so many words, to point out that this method could be used as the starting point for all quantum mechanics, and to suggest it as a practical way of doing quantum mechanical calculations.

Richard did just those things, I understand, in his 1942 dissertation, and then used the "path integral" or "sum over paths" approach in a great deal of his subsequent research. It was the basis, for example, of his way of arriving at the now standard covariant method of calculation in quantum field theory (which Ernst Stueckelberg reached in a different manner). That method is, of 
course, always presented in terms of "Feynman diagrams" such as the ones Dick later had painted on his van.

The sum-over-paths formulation is particularly convenient for integrating out one set of coordinates to concentrate on the remaining set. Thus the photon propagator in quantum electrodynamics is obtained ${ }^{1}$ by "integrating out" the photon variables, leaving electrons and positrons, both real and virtual, to interact by means of the covariant function $\delta\left(x^{2}\right)+\left(\pi \mathrm{i} x^{2}\right)^{-1}$.

In 1963 Feynman and his former student F. L. Vernon Jr, carrying further some research Ugo Fano had earlier done in a different way, showed how in a wide variety of problems of concern to laser physicists, condensed matter physicists and others of a practical bent, one can integrate out variables that are not of interest to throw light on the behavior of the ones that are kept. If initially the density matrix factors into one part depending on the interesting variables and another part depending on the rest, then the subsequent time development of the reduced density matrix for the interesting variables can be expressed in terms of a double path integral in which the coefficient of the initial reduced density matrix is $\exp \left[\mathrm{i}\left(S-S^{\prime}+W\right) / \hbar\right]$, where $S$ is the action along the path referring to the lefthand side of the density matrix, $S^{\prime}$ is the action along the path referring to the right-hand side of density matrix, and $W$ is the "influence functional," depending on both paths, that comes from integrating out all the uninteresting variables. Feynman and Vernon worked out a number of cases in detail, and subsequent research by A. O. Caldeira and Anthony Leggett, among others, further clarified some of the issues involved.

\section{Shedding light on quantum mechanics}

More recently, in the work of H. Dieter Zeh, of Erich Joos and of Wojciech Zurek and others, this line of research has thrown important light on how quantum mechanics produces decoherence, one of the conditions for the nearly classical behavior of familiar objects. For a planet, or even a dust grain, undergoing collisions with, for example, the photons of the $3-\mathrm{K}$ radiation, the imaginary part of the functional $W$ resulting from the integration over those quanta can yield, in $\exp (\mathrm{i} W / \hbar)$, a factor that decreases exponentially with some measure of the separation between the coordinate trajectory on the left side of the density matrix and that on the right. The density matrix can thus be constrained to remain nearly diagonal in the coordinates of the particle, giving rise to decoherence. If in addition the dust grain's inertia is large enough that the grain resists, for the most part, the disturbances of its trajectory caused by the quantum and thermal fluctuations of the background, and also large enough that the quantum spreading of the coordinate is slow, then the behavior of the grain's position operator will be nearly classical.

When an operator comes into correspondence with a nearly classical operator, then the first operator can be measured or observed. Thus work such as that of Feynman and Vernon has led not only to practical applications but also to a better understanding of how quantum mechanics produces the world with which we are familiar.

The path integral approach has proved in numerous situations to be a useful alternative to the conventional formulation of quantum mechanics in terms of operators in Hilbert space. It has many advantages besides the ease of integrating out, under suitable conditions, some of the variables. The path integral method, making use as it does of the action, can usually display in an elegant manner the invariances of the theory and can point the way toward exhibiting those invariances in a perturbation expansion. It is obviously a good approach for deriving the classical limit, and it can also be very helpful in semiclassical approximations, for example, in the description of tunneling. For certain effects, such as tunneling via instantons, it permits calculations that are highly nonperturbative in the usual sense. It is also particularly good for the global study of field configurations in quantum field theory, as it permits a straightforward discussion of topological effects.

Of course the conventional approach is superior for certain purposes, such as exhibiting the unitarity of the $S$ matrix and the fact that probabilities are not negative. Richard would never have contemplated, as he did around 1948, the consistent omission of all closed loops in quantum electrodynamics if he had been thinking in terms of a Hamiltonian formulation, where unitarity, which rules out such an omission, is automatic. (The impossible theory without closed loops could, by the way, realize the remarkable vision of Wheeler, which Richard said Wheeler once awakened him to explain: Not only are positrons electrons going backward in time, but all electrons and positrons represent the same electron going backward and forward, thus explaining why they all have the same absolute value of the electric charge!)

In any case, the path integral formulation remained merely a reformulation of quantum mechanics, equivalent to the usual formulation. I say "merely" because Richard, with his great talent for working out, sometimes in dramatically new ways, the consequences of known laws, was unnecessarily sensitive on the subject of discovering new ones. He wrote, in connection with the discovery of the universal vector and axial vector weak interaction in 1957: "It was the first time, and the only time, in my career that I knew a law of nature that nobody else knew. (Of course, it wasn't true, but finding out later that at least Murray Gell-Mann-and also [E. C. George] Sudarshan and [Robert] Marshak-had worked out the same theory didn't spoil my fun.)... It's the only time I ever discovered a new law."2

Thus it would have pleased Richard to know (and perhaps he did know, without my being aware of it) that there are now some indications that his $\mathrm{PhD}$ dissertation may have involved a really basic advance in physical theory and not just a formal development. The path integral formulation of quantum mechanics may be more fundamental than the conventional one, in that there is a crucial domain where it may apply and the conventional formulation may fail. That domain is quantum cosmology.

\section{Seeking rules for quantum gravity}

Of all the fields in fundamental physical theory, the gravitational field is picked out as controlling, in Einsteinian fashion, the structure of space-time. This is true even in a unified description of all the fields and all the particles of nature. Today, in superstring theory, we have the first 


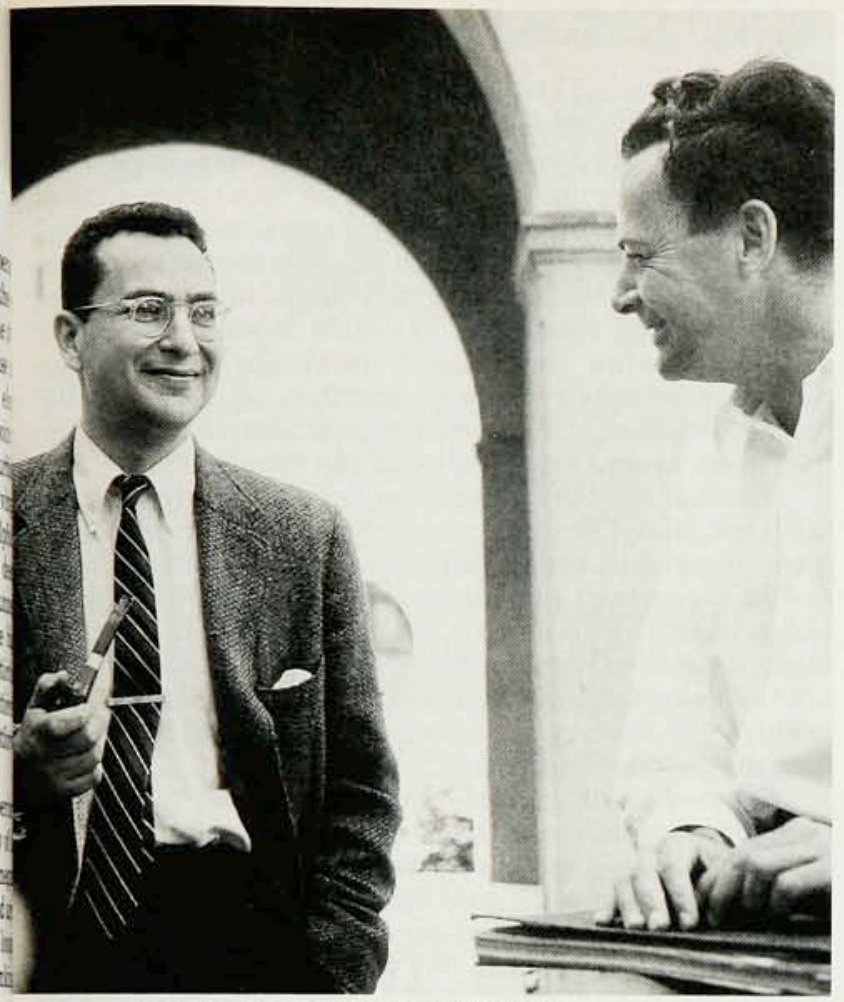

IOE MUNROE/CALIFORNIA INSTITUTE OF TECHNOLOGY

respectable candidate for such a theory, apparently finite in perturbation theory and describing, roughly speaking, an infinite set of local fields, one of which is the gravitational field linked to the metric of space-time. If all the other fields are dropped, the theory becomes an Einsteinian theory of gravitation.

Now the failure of the conventional formulation of quantum mechanics, if it occurs, is connected with the quantum mechanical smearing of space-time that is inevitable in any quantum field theory that includes Einsteinian gravitation.

If there is a dominant background metric for spacetime, especially a Minkowskian metric, and one is treating the behavior of small quantum fluctuations about the background (for example, the scattering of gravitons by gravitons), then the deep questions about space-time in quantum mechanics do not come to the fore.

Dick played a major part in working out the rules of quantum gravity in that approximation. It so happened that I was peripherally involved in the story of that research. We first discussed it when I visited Caltech during the Christmas vacation of 1954-55 and he was my host. (I was offered a job within a few days-such things would take longer now.) I had been interested in a similar approach, sidestepping the difficult cosmological issues, and when I found that he had made considerable progress I encouraged him to continue, to calculate one-loop effects and to find out whether quantum gravity was really a divergent theory to that order. He was always very suspicious of unrenormalizability as a criterion for rejecting theories, but he did pursue the research on and off. In 1960 he complained to me that he was having trouble. His covariant diagram method was giving results incompatible with unitarity. The imaginary part of the amplitude for a fourth-order process should be related directly to the product of a second-order amplitude and the complex conjugate of a second-order amplitude. That relation was failing.
In the 'Court of the Oak' at Caltech in 1959, Feynman and the author enjoy a relaxed moment.

I suggested that he try the analogous problem in Yang-Mills theory, a much simpler nonlinear gauge theory than Einsteinian gravitation. Richard asked what Yang-Mills theory was. (He must have forgotten, because in 1957 we worked out the coupling of the photon to the charged intermediate boson for the weak interaction and noticed that it was the right coupling for a Yang-Mills theory of those quanta.) Anyway, it didn't take long to teach him the rudiments of Yang-Mills theory, and he threw himself with renewed energy into resolving the contradiction. He found, eventually, that in the Lorentzcovariant formulation of either theory it was necessary to introduce some weird supplementary fields called "ghosts," and they have been used ever since, acquiring more and more importance. He described them at a meeting in Poland (in 1963, I think). Usually they are called "Faddeev-Popov ghosts" after L. D. Faddeev and V. N. Popov, who also studied them.

Thus Feynman was able to report in the 1960 s that Einsteinian gravitation was terribly divergent when interacting with electrons, photons or other particles. (The divergences in pure quantum gravitation theory turned out to be serious too, but that was shown much later, in the two-loop approximation, by two Caltech graduate students, Marc Goroff and Augusto Sagnotti.)

Those problems may be rectified by unification of all the particles and interactions, as they are in superstring theory. But we must still face up to the issues raised by the fact that the metric is up for quantum mechanical grabs and cannot in general be treated as a simple classical background plus small quantum fluctuations.

\section{Quantum cosmology}

Recently there has been great progress in thinking about the cosmological aspects of quantized Einsteinian gravitation. The work of Stephen Hawking and James Hartle, as well as Claudio Teitelboim, Alexander Vilenkin, Jonathan Halliwell and several others, has shown how the path integral method can probably deal with the situation and how it may be possible to generalize the method so as to describe not only the dynamics of the universe but also its initial boundary condition in terms of the classical action $S$. Furthermore, there are now, as I mentioned above, some indications that the conventional formulation of quantum mechanics may not be justifiable except to the extent that a background space-time emerges with small quantum fluctuations. Hartle in particular has emphasized such a possibility.

One crude way to see the argument is to express the wavefunction of the universe (which we assume to be in a pure state) as a path integral over all the fields in nature (for example, the infinity of local fields represented, roughly speaking, by the superstring), reserving the integral over the metric $g_{\mu v}$ for last. The total action $S$ can be represented as the Einstein action $S_{\mathrm{G}}$ for pure gravitation plus the actions $S_{\mathrm{M}}$ for all the other, "matter" fields, including their coupling to gravitation. We have, then, crudely,

Amplitude $=$

$$
\int \mathscr{D} g_{\mu v} \exp \frac{i S_{\mathrm{G}}}{\hbar} \times \int \mathscr{D} \text { (everything else) } \exp \frac{\mathrm{i} S_{\mathrm{M}}}{\hbar}
$$

For the moment, suppose only $g_{\mu v}$ configurations corre- 
sponding to a simple topology for space-time are allowed. Before the integration over $g_{\mu v}$ is performed, there is a definite space-time, with the possibility of constructing well-defined space-like surfaces in a definite succession described by a time-like variable. There is an equivalent Hilbert-space formalism; we have unitarity (conservation of positive probability); and we can have conventional causality (it corresponds in the Hilbert-space formulation to the requirement of time ordering of operators in the formula for probabilities).

Now, when the integral over $g_{\mu v}$ is done, it is no longer clear that any of that machinery remains, since we are integrating over the structure of space-time and once the integral is performed it is hard to point to space-like surfaces or a succession described by a timelike variable. Of course it may be possible to construct a Hilbert-space formulation, with unitarity and causality, in some new way, perhaps employing a new, external time variable of some kind (what Feynman liked to call a fifth wheel), but it is by no means certain that such a program can be carried out.

At this stage, we may admit the possibility of summing over all topologies of space-time (or of the corresponding space-time with a Euclidean metric). If that is the correct thing to do, then we are immediately transported into the realm of baby universes and wormholes, so beloved of Stephen Hawking and now so fashionable, in which it seems to be demonstrable that the cosmological constant vanishes. In that realm the path integral method appears able to cope, and it remains to be seen to what extent the conventional formulation of quantum mechanics can keep up.

For Richard's sake (and Dirac's too), I would rather like it to turn out that the path integral method is the real foundation of quantum mechanics and thus of physical theory. This is true despite the fact that, having an algebraic turn of mind, I have always personally preferred the operator approach, and despite the added difficulty, in the absence of a Hilbert-space formalism, of interpreting the wavefunction or density matrix of the universe (already a bit difficult to explain in any case, as anyone attending my classes will attest). If notions of transformation theory, unitarity and causality really emerge from the mist only after a fairly clear background metric appears (that metric itself being the result of a quantum mechanical probabilistic process), then we may have a little more explaining to do. Here Dick Feynman's talents and clarity of thought would have been a help.

\section{Turning things around}

Richard, as is well known, liked to look at each problem, important or unimportant, in a new way- "turning it around," as he would say. He told how his father, who died when he was young, taught him to do that. This approach went along with Richard's extraordinary efforts to be different, especially from his friends and colleagues.

Of course any of us engaged in creative work, and in fact anyone having a creative idea even in everyday life, has to shake up the usual patterns in some way in order to get out of the rut (or the basin of attraction!) of conventional thinking, dispense with certain accepted but wrong notions, and find a new and better way to formulate some problem. But with Dick, "turning things around" and being different became a passion.

The result was that on certain occasions, in scientific work or in ordinary living, when an imaginative new way of looking at things was needed, he could come up with a remarkably useful innovation. But on many other occa- sions, when the usual way of doing business had its virtues, he was not the ideal person to consult. Remember his television appearance in which he made fun of the daily habit of brushing one's teeth? (And he didn't even suggest flossing!) Or take his occasional excursions into far-out political choices in the 1950 s, during his second marriage Those certainly set him off from most of his friends. But one day during that time, he called me and sheepishly admitted having voted for a particularly outrageous candidate for statewide office-and then asked me if in the future I would check over such names beforehand and tell him when he was really going off the deep end!

None of the aberrations mentioned here changes the fact that Dick Feynman was a most inspiring person. I have referred to his originality and straightforwardness and to his energy, playfulness and vitality. All of those characteristics showed up in his work and also in the other facets of his life. Indeed, that vitality may be related to the kind of biological (and probably psychological) vitality that enabled him to resist so remarkably and for so long the illness to which he finally succumbed.

When I think of him now, it is usually as he was during that first decade that we were colleagues, when we were both young and everything seemed possible. We phoned each other with good ideas and crazy ones, with serious messages and farcical gags. We yelled at each other in front of the blackboard. We taught stewardesses to say "quark-quark scattering" and "quark-antiquark scattering." We delivered a peacock to the bedroom of our friend Jirayr Zorthian on his birthday, while our wives distracted him. We argued about everything under the Sun.

Later on, we drifted apart to a considerable extent, but I was aware, all the time we were colleagues, that if a really profound question in science came up, there would be fun and profit in discussing it with Dick. Even though on many occasions during the last 20 years, I passed up the opportunity to talk with him in such a case, I knew that I could do so, and that made a great difference.

Besides, I did not always pass it up. For example, during the last few months and even weeks of his life, we kept up a running discussion of one of the most basic subjects, the role of "classical objects" in the interpretation of quantum mechanics. We thus resumed a series of conversations on that topic that we had begun a quarter of a century earlier. In between 1963-64 and 1987 those talks about quantum mechanics were rare, but there was at least one remarkable occasion during the last few of those years. Richard sat in on one of my classes on the meaning of quantum mechanics, interrupting from time to time. He did not, however, object to what I was saying; rather, he reinforced the points I was making. The students must have been delighted as they heard the same arguments made by both of us in a kind of counterpoint.

It is hard for me to get used to the fact that now, when I have a deep issue in physics to discuss with someone, Dick Feynman is no longer around.

I should like to thank James B. Hartle for many instructive conversations about quantum mechanics and the path integral method in quantum cosmology.

\section{References}

1. R. P. Feynman, Phys. Rev. 76, 749, 769 (1949).

2. R. P. Feynman, "Surely You're Joking, Mr. Feynman!' Adventures of a Curious Character, Bantam, New York (1986), p. 229. See also M. Gell-Mann, in Proc. Int. Mtg. on the History of Scientific Ideas, M. G. Doncel et al., eds., Bellaterra, Barcelona (1987), p. 474. 